\title{
Effect of gold nanoparticles size on light scattering for thin film amorphous-silicon solar cells
}

\author{
Kazi Islam $^{\mathrm{a}}$, Aaesha Alnuaimi ${ }^{\mathrm{a}}$, Enes Battal ${ }^{\mathrm{b}}$, Ali Kemal Okyay ${ }^{\mathrm{b}}$, Ammar Nayfeh ${ }^{\mathrm{a}, *}$ \\ ${ }^{a}$ Institute Center for Future Energy Systems (iFES), Department of Electrical Engineering and Computer Science (EECS), Masdar Institute of Science \\ and Technology, Abu Dhabi, United Arab Emirates \\ ${ }^{\mathrm{b}}$ Department of Electrical and Electronics Engineering, UNAM-National Nanotechnology Research Center, Institute of Materials Science \\ and Nanotechnology, Bilkent University, 06800 Ankara, Turkey
}

Received 3 October 2013; received in revised form 8 February 2014; accepted 11 February 2014

Available online 5 March 2014

Communicated by: Associate Editor Frank Nuesch

\begin{abstract}
In this work, the effect of gold $(\mathrm{Au})$ nanoparticles on the performance of a-Si:H solar cells is investigated experimentally. The solar cell stack is grown on a highly doped p-type $\mathrm{Si}$ wafer and consists of $20 \mathrm{~nm}$ heavily doped p-type a-Si, $500 \mathrm{~nm}$ undoped a-Si, $20 \mathrm{~nm}$ heavily doped n-type a-Si and finally $80 \mathrm{~nm}$ Indium Tin Oxide (ITO) on the top. Au nanoparticles of 10, 20, 50, 80, 100, 200 and 400 nm are spin coated on top of the ITO before metallization. The plasmonic effect of the Au nanoparticles allows for additional scattering at the surface thus reducing the overall reflectivity. The larger the nanoparticle size the more scattering is obtained and the median reflectivity drops from about $23 \%$ to $18 \%$. The results show an increase in the short-circuit current density $\left(J_{s c}\right)$ and efficiency with increasing nanoparticle size. The $J_{s c}$ increases from 9.34 to $10.1 \mathrm{~mA} / \mathrm{cm}^{2}$. In addition, the efficiency increases from $4.28 \%$ to $5.01 \%$.
\end{abstract}

(C) 2014 Elsevier Ltd. All rights reserved.

Keywords: Nanoparticles; Photovoltaics; Plasmonics; Thin film; Solar cells; Amorphous Si

\section{Introduction and motivation}

Effective light trapping mechanisms are important for the improved performance of thin film solar cells. For enhanced absorption in thin film solar cells, different approaches such as surface texturing and back reflectors have been described (Berginski et al., 2008; Ferry et al., 2009). More recently, nanotechnology has been used with plasmonic light trapping of metal nanoparticles like gold $(\mathrm{Au})$ and silver (Ag) (Atwater and Polman, 2010; Catchpole and Polman, 2008; Akimov et al., 2009). For incorporating metal nanoparticles into solar cells, different methods have been

\footnotetext{
* Corresponding author.

E-mail address: anayfeh@masdar.ac.ae (A. Nayfeh).
}

established that includes island annealing and colloidal metal particles (Beck et al., 2009; Derkacs et al., 2006). Also, some numerical models have been developed to understand the plasmonic effect (Akimov et al., 2009). Plasmonic nanoparticles have been explored extensively for dye-sensitized solar cells (DSSC) (Lin et al., 2012) and a nanoparticle-size dependent study similar study to ours but for DSSC has been published in Deepa et al. (2012). Though most of the publications in this field have presented data for back-scattering by implementing the nanoparticles in the back side of the solar cells, a recent study showed insightful results of placing Ag nanoparticles on the top surface of solar cells (Lenzmann et al., 2013).

Surface plasmons are collective oscillations of the free charges at a metal boundary. Metals support surface 
plasmons, either localized as for metal nanoparticles or propagating in case of planar metal surfaces. By controlling the size and shape of the metallic nanostructures, the surface plasmon resonance or plasmon propagating properties can be varied. Since the surface resonances of metals are mostly in the visible or in the infrared region of the electromagnetic spectrum, they are of particular interest for photovoltaic application (Bohren and Huffman, 2008). Two prominent mechanisms can explain the contribution of metallic nanoparticles based on application: scattering mechanism and the near-field localization effect (Temple et al., 2009). Photocurrent enhancement by metal nanoparticles on the top surface of solar cells can be explained by the light scattering mechanism. Metal nanoparticles are strong scatterers of light at wavelengths near their resonant frequency (Lenzmann et al., 2013).

\section{Structure and fabrication process: spin-coating gold nanoparticles}

Typically nanoparticles are placed on the bottom of the solar cell to enhance back scattering as well as back reflection. In this work, the effect of gold nanoparticles size placed on the top surface of thin film a-Si:H solar cells is studied.

In order to study the effect of the gold nanoparticles, an a-Si:H solar cell is fabricated. Fig. 1 shows the structure of the fabricated a-Si:H solar cell. The solar cell stack is grown on a p+ type Si wafer and consists of $20 \mathrm{~nm}$ heavily doped p-type a-Si, $500 \mathrm{~nm}$ undoped a-Si, $20 \mathrm{~nm}$ heavily doped n-type a-Si and finally $80 \mathrm{~nm}$ Indium Tin Oxide (ITO) on the top. The $\mathrm{p}+\mathrm{Si}$ substrate serves as the back contact, and does not contribute significantly to the carrier generation. The $\mathrm{n}+$, intrinsic and $\mathrm{p}+$ layers of aSi:H were grown using Plasma Enhanced Chemical Vapor Deposition (PECVD) tool in a single run on the $\mathrm{P}+\mathrm{Si}$ substrate. Then $80 \mathrm{~nm}$ of ITO was sputtered on the top layer using RF sputtering tool (http://www.mvsystemsinc.com/researchand-development-services, 2013). More details of the solar cell fabrication process flow can be found in Hadi et al.

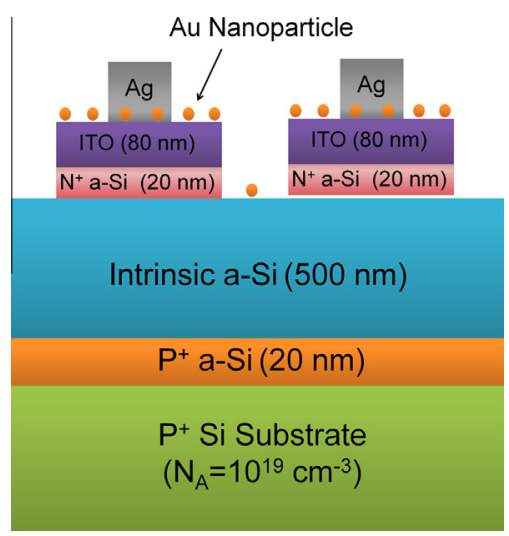

Fig. 1. Schematic cross-section of the fabricated ITO/a-Si:H solar cell with $\mathrm{Au}$ nanoparticles deposited on the top surface. (Figure not to scale).
(2011, 2012), Alnuaimi et al. (2013). The gold nanoparticles were spin-coated on top of the ITO.

There are various ways to deposit the nanoparticles as mentioned earlier including island forming by annealing, drop-casting, dip coating and spin coating. In this work, colloidal gold nanoparticles dispersed in Phosphate Buffered Saline (PBS) solution were used (http://www.sigmaaldrich.com/materials-science/nanomaterials/gold-nanoparticles, 2013). In order to study the effect of the gold nanoparticles size, seven different diameter-size i.e., $10 \mathrm{~nm}, 20 \mathrm{~nm}$, $50 \mathrm{~nm}, 80 \mathrm{~nm}, 100 \mathrm{~nm}, 200 \mathrm{~nm}$ and $400 \mathrm{~nm}$ particle sizes were investigated. The spin coating recipe was optimized to obtain uniform distribution as well as optimum concentration of particles on the surface. Several spin-coating recipes are investigated and the most optimized spin-coating recipe is found to be $2000 \mathrm{rpm}$ speed, $1000 \mathrm{rpm}$ acceleration and spinning for $60 \mathrm{~s}$. Fig. 2 is an SEM image of the top surface showing the gold nanoparticles on the ITO. The surface coverage by the nanoparticles is approximately calculated from the SEM images and is found to be $0.07854 \%$ for the $100 \mathrm{~nm}$ nanoparticles with a variance of $0.016 \%$ based on different locations of the cell surface. We assume the surface coverage percentage remains in close vicinity for different nanoparticle sizes since number of nanoparticles per unit volume of the solution decreases with increasing nanoparticle sizes, as mentioned in the supplier's data sheet. For example, there are $5.98 \times 10^{12}$ nanoparticles $/ \mathrm{ml}$ of solution for $10 \mathrm{~nm} \mathrm{Au}$ particles whereas the number of nanoparticles for $100 \mathrm{~nm} \mathrm{Au}$ particles is $3.84 \times 10^{9}$ per $\mathrm{ml}$ of the PBS

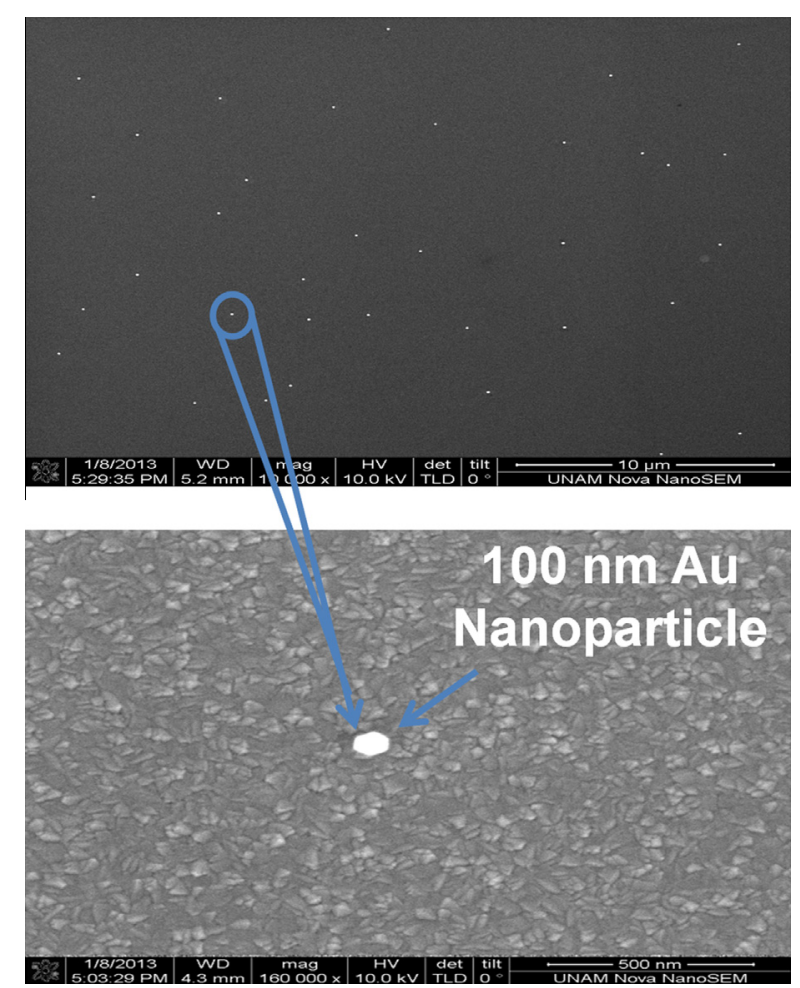

Fig. 2. SEM image of the top surface of the fabricated $n-i-p$ a-Si:H solar cell with $100 \mathrm{~nm} \mathrm{Au}$ nanoparticles on the ITO surface. 
solution (http://www.sigmaaldrich.com/materials-science/ nanomaterials/gold-nanoparticles, 2013). In all cases, we used about $5 \mathrm{ml}$ of solution containing nanoparticles for spinning and the percentage of the surface covered by different sizes of nanoparticles in each cases is assumed to be in a close range, though further study is required to confirm this.

After the nanoparticles coating, $100 \mathrm{~nm}$ of silver was deposited using Thermal Physical Vapor Deposition (PVD) tools and lift-off technique was used to form the finger contacts. Three different cell area-sizes were fabricated i.e. $1 \mathrm{~cm} \times 1 \mathrm{~cm}, \quad 0.5 \mathrm{~cm} \times 0.5 \mathrm{~cm}$ and $0.25 \mathrm{~cm} \times 0.25 \mathrm{~cm}$. Unless otherwise stated, solar cells of size $0.25 \mathrm{~cm} \times 0.25 \mathrm{~cm}$ are presented in this work.

\section{Experimental results and analysis}

This section presents the mean values for all experimental data. Fig. 3 shows the $J-V$ curve for the reference cell without nanoparticles and cells with $10 \mathrm{~nm}$ and $400 \mathrm{~nm}$ nanoparticles. The $V_{o c}$ remains $0.89 \mathrm{~V}$ and does not change significantly with nanoparticles while the $J_{s c}$ improves with larger nanoparticles size. Fig. 4 plots $J_{s c}$ vs. nanoparticles size. The $J_{s c}$ increases from 9.34 to $10.1 \mathrm{~mA} / \mathrm{cm}^{2}$. Fig. 5 plots the median reflectance across all wavelengths vs. nanoparticles size. The median reflectance drops from $23.19 \%$ with no nanoparticles to less than $20 \%$ for all nanoparticles sizes. The drop shows the improved surface scattering due to the gold nanoparticles. The efficiency increases with increasing nanoparticles size due to the increase in the $J_{s c}$, from $4.28 \%$ to $5.1 \%$. The fill factor (FF) increases from $51 \%$ for the reference cell up to $56 \%$ for the gold nanoparticles based cells of all sizes. It should be noted that the FF is independent of particle size but does tend to increase slightly with increasing size. This may be due to a reduction in contact resistance with the addition of metal nanoparticles, but further studies are to be done to confirm this.

The External Quantum Efficiency (EQE) of the fabricated devices shows an improved spectral response with increasing nanoparticles size. The peak EQE is $59 \%$ for the cell with $400 \mathrm{~nm}$ nanoparticles while the reference cell has 53\% peak EQE as shown in Fig. 6. Table 1 summarizes

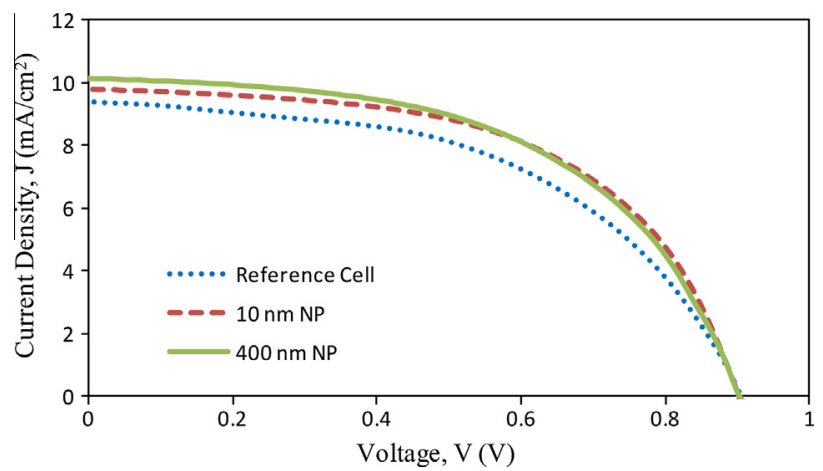

Fig. 3. Measured $J-V$ characteristics of $n-i-p$ a-Si:H reference cell without nanoparticles and cells with $10 \mathrm{~nm}$ and $400 \mathrm{~nm}$ Au nanoparticles.

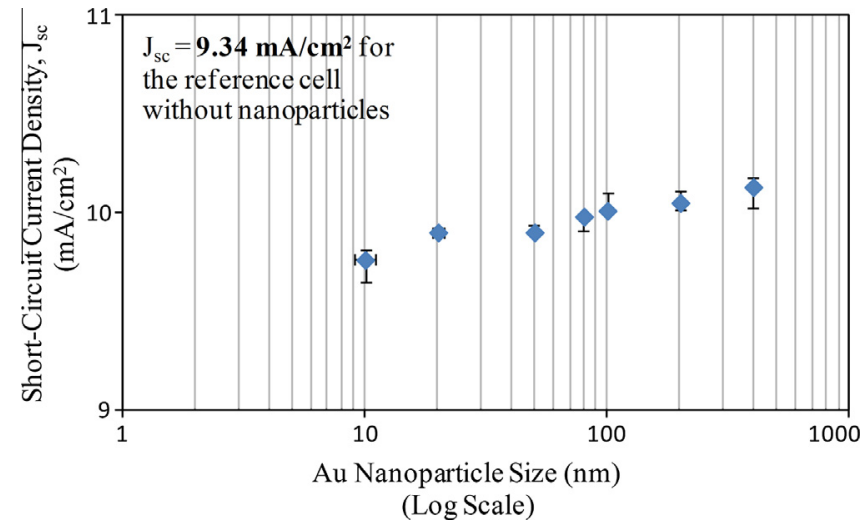

Fig. 4. $J_{s c}$ vs. Au nanoparticle size (plotted in log scale) for $\mathrm{Au}$ nanoparticle enhanced plasmonic $\mathrm{n}-\mathrm{i}-\mathrm{p}$ a-Si:H solar cells.

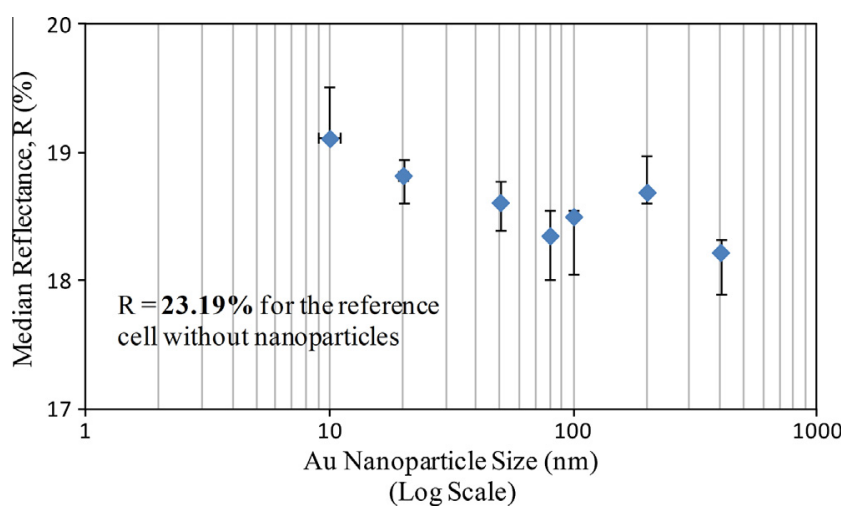

Fig. 5. Median reflectance vs. Au nanoparticle size (plotted in log scale) for Au nanoparticle enhanced plasmonic $\mathrm{n}-\mathrm{i}-\mathrm{p}$ a-Si:H solar cells.

the main findings of this work with statistical analysis, listing the $J_{s c}$ and efficiency of the solar cells with respect to the gold nanoparticles size. The $J_{s c}$ increases by $8.46 \%$ with the introduction of nanoparticles for the best case and the efficiency improves by $17.06 \%$ with nanoparticles for the best case as well. Both the $J_{s c}$ and efficiency tend to improve with larger nanoparticles sizes.

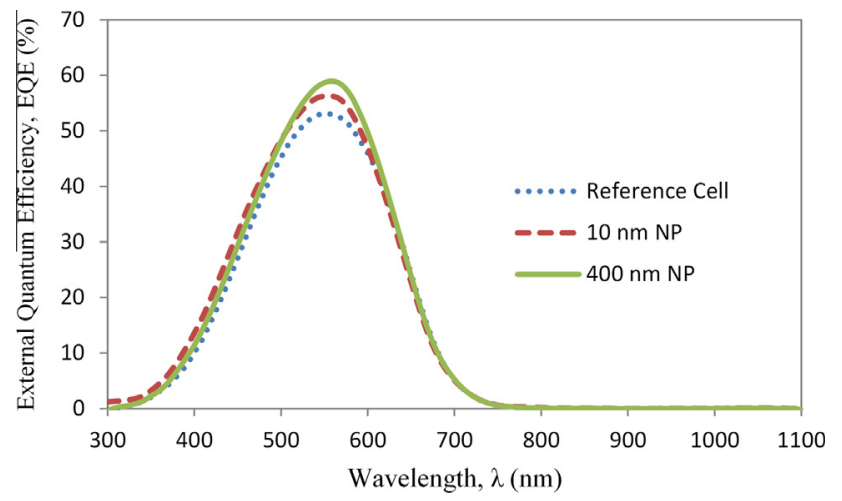

Fig. 6. Measured external quantum efficiency of $n-i-p$ a-Si:H reference cell without nanoparticles and cells with $10 \mathrm{~nm}$ and $400 \mathrm{~nm} \mathrm{Au}$ nanoparticles. 
Table 1

Summary of results.

\begin{tabular}{|c|c|c|c|c|}
\hline & $V_{o c}(\mathrm{~V})$ & $J_{s c}\left(\mathrm{~mA} / \mathrm{cm}^{2}\right)$ & $\eta(\%)$ & Median $R(\%)(400-1100 \mathrm{~nm})$ \\
\hline \multicolumn{5}{|c|}{ Reference cell (without nanoparticles) } \\
\hline Maximum & 0.89 & 9.34 & 4.28 & 23.92 \\
\hline Average & 0.89 & 9.34 & 4.27 & 23.35 \\
\hline Minimum & 0.89 & 9.3 & 4.25 & 22.95 \\
\hline Standard deviation & 0 & 0.05 & 0.01 & 0.51 \\
\hline \multicolumn{5}{|l|}{$A u N P$ size $=10 \mathrm{~nm}$} \\
\hline Maximum & 0.89 & 9.81 & 4.84 & 19.52 \\
\hline Average & 0.89 & 9.74 & 4.79 & 19.24 \\
\hline Minimum & 0.89 & 9.74 & 4.71 & 19.1 \\
\hline Standard deviation & 0 & 0.08 & 0.05 & 0.24 \\
\hline \multicolumn{5}{|l|}{$\mathrm{Au} N P$ size $=20 \mathrm{~nm}$} \\
\hline Maximum & 0.89 & 9.9 & 4.9 & 18.95 \\
\hline Average & 0.89 & 9.90 & 4.89 & 18.79 \\
\hline Minimum & 0.89 & 9.89 & 4.88 & 18.61 \\
\hline Standard deviation & 0 & 0.01 & 0.01 & 0.17 \\
\hline \multicolumn{5}{|l|}{$A u N P$ size $=50 \mathrm{~nm}$} \\
\hline Maximum & 0.89 & 9.94 & 4.98 & 18.78 \\
\hline Average & 0.89 & 9.91 & 4.93 & 18.59 \\
\hline Minimum & 0.89 & 9.89 & 4.9 & 18.39 \\
\hline Standard deviation & 0 & 0.03 & 0.03 & 0.20 \\
\hline \multicolumn{5}{|l|}{$\mathrm{Au} N P$ size $=80 \mathrm{~nm}$} \\
\hline Maximum & 0.89 & 9.99 & 4.95 & 18.55 \\
\hline Average & 0.89 & 9.96 & 4.93 & 18.30 \\
\hline Minimum & 0.89 & 9.91 & 4.9 & 18.01 \\
\hline Standard deviation & 0 & 0.04 & 0.02 & 0.27 \\
\hline \multicolumn{5}{|l|}{$\mathrm{Au} N P$ size $=100 \mathrm{~nm}$} \\
\hline Maximum & 0.89 & 10.1 & 5 & 18.55 \\
\hline Average & 0.89 & 10.04 & 4.99 & 18.37 \\
\hline Minimum & 0.89 & 10.01 & 4.97 & 18.05 \\
\hline Standard deviation & 0 & 0.05 & 0.01 & 0.28 \\
\hline \multicolumn{5}{|l|}{$\mathrm{Au} N P$ size $=200 \mathrm{~nm}$} \\
\hline Maximum & 0.89 & 10.11 & 5.01 & 18.98 \\
\hline Average & 0.89 & 10.06 & 5.01 & 18.76 \\
\hline Minimum & 0.89 & 10.01 & 5 & 18.6 \\
\hline Standard deviation & 0 & 0.05 & 0.00 & 0.20 \\
\hline \multicolumn{5}{|l|}{ Au NP size $=400 \mathrm{~nm}$} \\
\hline Maximum & 0.89 & 10.18 & 5.05 & 18.32 \\
\hline Average & 0.89 & 10.11 & 5.02 & 18.14 \\
\hline Minimum & 0.88 & 10.02 & 5 & 17.89 \\
\hline Standard deviation & 0 & 0.08 & 0.02 & 0.23 \\
\hline
\end{tabular}

\section{Physics of plasmonic enhancement}

In order to understand the physics of the effect of gold nanoparticles size a mathematical model is used. A point dipole model can describe the absorption and scattering of the incoming light by the nanoparticles for particles with diameters well below the wavelength the incoming light. The scattering and absorption cross-sections are given by the following equations (Lenzmann et al., 2013):

$C_{\text {scat }}=\frac{1}{6 \pi}\left(\frac{2 \pi}{\lambda}\right)^{4}|\alpha|^{2}$

$C_{a b s}=\frac{2 \pi}{\lambda} \operatorname{Im}[\alpha]$ where

$\alpha=3 V\left[\frac{\varepsilon_{p} / \varepsilon_{m}-1}{\varepsilon_{p} / \varepsilon_{m}+2}\right]$

is the polarizability of the particle. Here $V$ is the particle volume, $\varepsilon_{p}$ is the dielectric function of the particle and $\varepsilon_{m}$ is the dielectric function of the embedding medium. From (3), its quite obvious that at $\varepsilon_{p}=-2 \varepsilon_{m}$ the particle polarizability will become very large and surface plasmon resonance occurs.

At the surface plasmon resonance, the scattering crosssection exceeds the geometrical cross section of the nanoparticles e.g. silver nanoparticles in air has a scattering cross-section that is approximately ten times the crosssectional area of the particle at the resonant frequency 


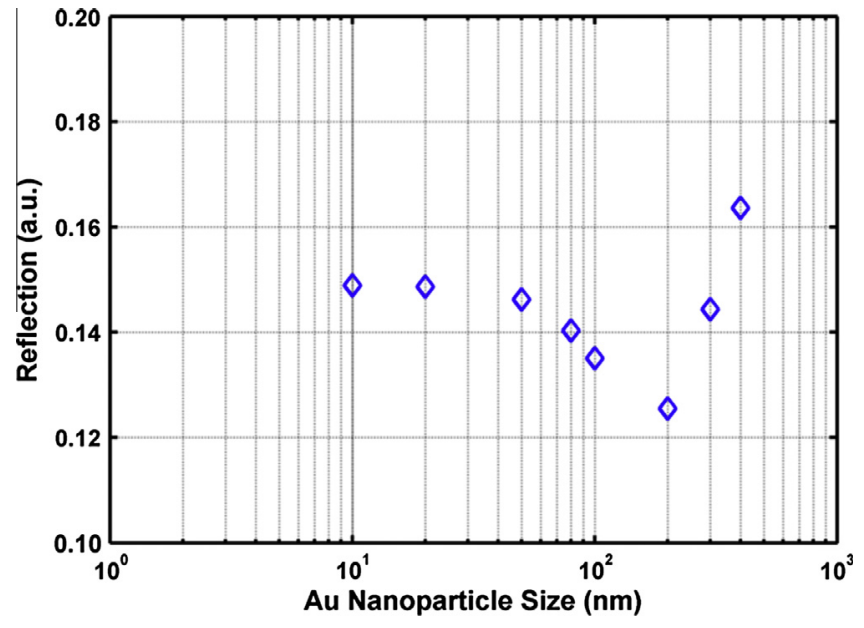

Fig. 7. Simulated reflection vs. Au nanoparticle size (plotted in log scale) for Au nanoparticle enhanced plasmonic solar cell.

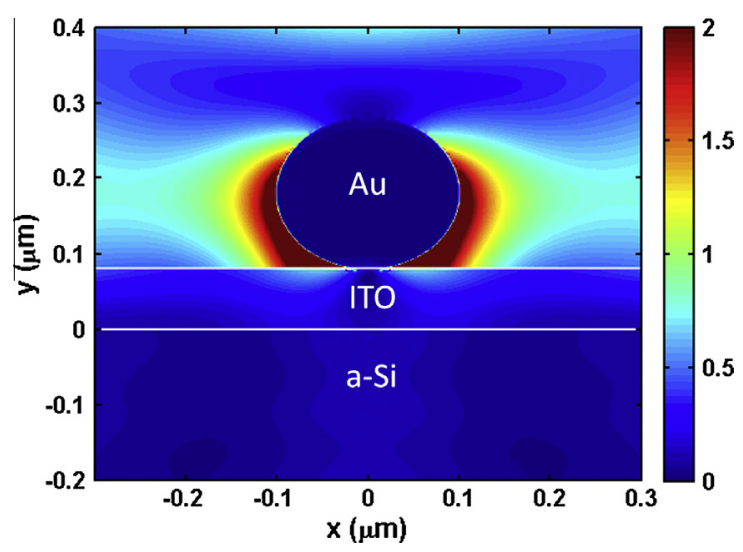

Fig. 8. Simulated electric field intensity profile for $200 \mathrm{~nm}$ spherical Au nanoparticles.

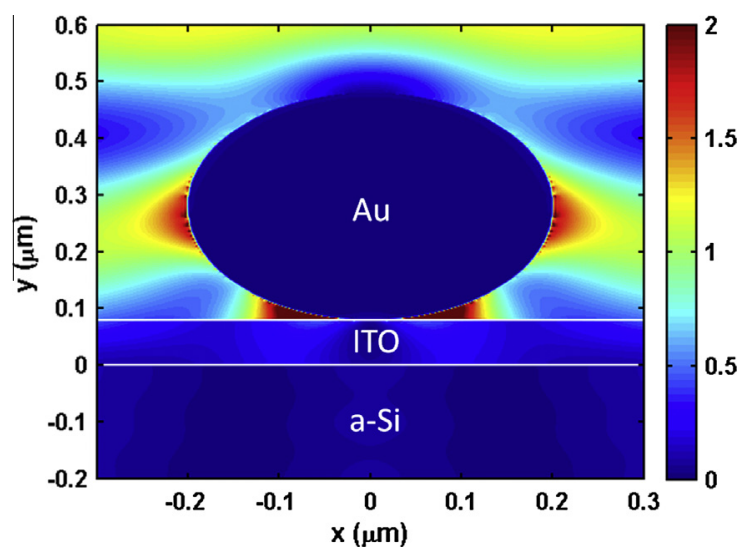

Fig. 9. Simulated electric field intensity profile for $400 \mathrm{~nm}$ spherical $\mathrm{Au}$ nanoparticles.

(Soller and Hall, 2002). In such a case, a substrate covered with a $10 \%$ areal density of nanoparticles can fully absorb and scatter the incoming light, to first-order (Soller and Hall, 2002; Catchpole and Pillai, 2006). For light trapping it is important that scattering is more efficient than absorp- tion, a condition that is met for comparatively larger nanoparticles as can be seen from (1) and (2) Catchpole and Polman, 2008. Nanoparticles size, shape and distribution over the surface will determine the effect of the nanoparticles on the performance of the photovoltaic device as a whole (Kelly et al., 2003).

In addition, the reflection from device surface in the presence of the Au nanoparticles is calculated by 2-dimensional finite difference time-domain (FDTD) method using FDTD Solutions from Lumerical Inc. For computational simplicity, monosized and spherical particles are used which are assumed to be electrically and optically isolated. A mesh size of $2 \mathrm{~nm}$ is used in all simulations and the illumination is from the top. Fig. 7 shows the results of computed reflection as a function of nanoparticle size which shows a similar trend to measured reflection vs. nanoparticle size. The reflection units are arbitrary as the surface coverage of metal nanoparticles is not taken into account. The computed electric field intensity profile, for a nanoparticle size of $200 \mathrm{~nm}$, illuminated with $800 \mathrm{~nm}$ wavelength light, is shown in Fig. 8 and exhibits a clear dipole characteristics. Fig. 9, on the other hand, shows that for a nanoparticle size of $400 \mathrm{~nm}$ additional plasmonic modes emerges surrounding the nanoparticle. This is attributed to the fact that the quastatic electric field assumption is weakened as the nanoparticle size becomes comparable to the wavelength of light.

\section{Conclusions}

In summary, the effect of gold nanoparticles size on the light scattering for a-Si:H thin film solar cells was studied. The larger the nanoparticles are more light scattering is achieved. The results show an increase in $J_{s c}$, efficiency and spectral response with the gold nanoparticles. Moreover, the results highlight a promising and simple enhancement for future thin film solar cells using nanoparticles.

\section{Acknowledgements}

We would like to thank the students and the technical staff of UNAM at Bilkent University, Turkey for their help with the fabrication. We gratefully acknowledge financial support for this work provided by the Masdar Institute of Science and Technology.

\section{References}

Akimov, Y.A., Ostrikov, K., Li, E.P., 2009. Surface plasmon enhancement of optical absorption in thin-film silicon solar cells. Plasmonics $4.2,107-113$.

Akimov, Y.A. et al., 2009. Enhancement of optical absorption in thin-film solar cells through the excitation of higher-order nanoparticle plasmon modes. Opt. Express 17.12, 10195-10205.

Alnuaimi, A., Islam, K., Nayfeh, A., 2013. Reduction of interface traps at the amorphous-silicon/crystalline-silicon interface by hydrogen and nitrogen annealing. Sol. Energy 98, 236-240.

Atwater, H.A., Polman, Albert, 2010. Plasmonics for improved photovoltaic devices. Nat. Mater. 9.3, 205-213. 
Beck, F.J. et al., 2009. Tunable light trapping for solar cells using localized surface plasmons. J. Appl. Phys. 105 (11), 114310.

Berginski, M. et al., 2008. Recent development on surface-textured ZnO: Al films prepared by sputtering for thin-film solar cell application. Thin Solid Films 516 (17), 5836-5841.

Bohren, C.F, Huffman, D.R., 2008. Absorption and Scattering of Light by Small Particles. Wiley.com.

Catchpole, K.R., Pillai, S., 2006. Absorption enhancement due to scattering by dipoles into silicon waveguides. J. Appl. Phys. 100 (4), 044504.

Catchpole, K.R., Polman, Albert, 2008. Plasmonic solar cells. Opt. Express 16.26, 21793-21800.

Deepa, K.G., Lekha, P., Sindhu, S., 2012. Efficiency enhancement in DSSC using metal nanoparticles: a size dependent study. Sol. Energy 86 (1), 326-330.

Derkacs, D. et al., 2006. Improved performance of amorphous silicon solar cells via scattering from surface plasmon polaritons in nearby metallic nanoparticles. Appl. Phys. Lett. 89, 093103.

Ferry, V.E. et al., 2009. Improved red-response in thin film a-Si:H solar cells with soft-imprinted plasmonic back reflectors. Appl. Phys. Lett. 95 (18), 183503.

Hadi, S.A. et al., 2011. Thin film a-Si/c-Si $1_{1-x} \mathrm{Gex} / \mathrm{c}-\mathrm{Si}$ heterojunction solar cells: design and material quality requirements. ECS Trans. 41 (4), 3-14.
Hadi, S.A. et al., 2012. Thin film a-Si/c-Si $i_{1-x}$ Ge x/c-Si heterojunction solar cells with Ge content up to $56 \%$. 38th IEEE Photovoltaic Specialists Conference (PVSC)2012. IEEE.

http://www.mvsystemsinc.com/research-and-development-services. (Accessed 28.07.13).

http://www.sigmaaldrich.com/materials-science/nanomaterials/gold-nano particles.html. (Accessed 13.06.13).

Kelly, K.L. et al., 2003. The optical properties of metal nanoparticles: the influence of size, shape, and dielectric environment. J. Phys. Chem. B $107.3,668-677$.

Lenzmann, F.O. et al., 2013. Plasmonic light-trapping in a-Si:H solar cells by front-side Ag nanoparticle arrays: a benchmarking study. Phys. Status Solidi (a).

Lin, Su-Jien et al., 2012. Plasmon-enhanced photocurrent in dye-sensitized solar cells. Sol. Energy 86.9, 2600-2605.

Soller, B.J., Hall, D.G., 2002. Scattering enhancement from an array of interacting dipoles near a planar waveguide. JOSA B 19 (10), 2437-2448.

Temple, T.L. et al., 2009. Influence of localized surface plasmon excitation in silver nanoparticles on the performance of silicon solar cells. Sol. Energy Mater. Sol. Cells 93 (11), 1978-1985. 